\title{
La gestion des plaintes, une réelle opportunité de gestion de la qualité des soins
}

\section{Complaints management, a real opportunity of care quality management}

\author{
F. Thys \\ (C) SFMU et Springer-Verlag France 2011
}

Dans ce numéro des Annales françaises de médecine d'urgence, Anne Raynaud-Lambinet et ses collaborateurs [1] publient une analyse des lettres de plaintes adressées à leur service sur une période de cinq ans. Ce travail est un signal très positif pour notre spécialité, car il atteste d'une maturité croissante autorisant la mise en perspective et en questionnement de la pratique médicale dans ce contexte particulier. En effet, les spécificités respectives de l'exercice de la médecine d'urgence et de la morbidité ressentie des patients qui la sollicitent exposent un tel lieu de soins à de nombreuses frustrations et critiques tant de la part des soignés que des autres acteurs soignants internes ou externes.

L'opportunité est donc réelle d'utiliser un indicateur tel que l'analyse systématique des plaintes dans nos évaluations de pratiques. Toutefois, la démarche est incomplète, si elle omet de s'intégrer dans une logique plus large de qualité et de sécurité du patient. Une telle omission occulte parfois le fait que les plaintes ne reflètent pas forcément l'ensemble des dysfonctionnements structurels d'un service mais n'en sont que la timide révélation par un regard extérieur. Il faut avoir le courage comme soignant de considérer chaque plainte comme un signal d'alarme potentiel sur une pratique défaillante. Bien sûr, les institutions hospitalières et leur impact sur la santé individuelle peuvent représenter naturellement des lieux de créations et d'expressions de conflits. Il est opportun de saisir l'importance d'une mutation de point de vue pour quitter définitivement le seul mode défensif en organisant une information claire du patient, une gestion systématique des plaintes écrites et téléphoniques et, enfin, en obtenant qu'une structure de médiation soit organisée soit au niveau hospitalier ou mieux encore du service.

L'information du patient sur l'existence d'une charte de ses droits en termes de recours et d'adressage des plaintes

\section{F. Thys $(\square)$}

Service des urgences, cliniques universitaires Saint-Luc, université catholique de Louvain, avenue Hippocrate 10, B-1200 Bruxelles, Belgique

e-mail : frederic.thys@uclouvain.be peut faire craindre à certains un phénomène d'induction de celles-ci. Dans la pratique, il n'est pas évident que cela soit le cas. Ainsi, en Belgique, une loi sur la « responsabilité médicale sans faute » est entrée en vigueur en avril 2010, elle permet à un patient, qui a subi un dommage à la suite d'une intervention médicale, d'obtenir une indemnisation (financière) $\mathrm{du}$ Fonds des accidents médicaux, sans plus même devoir prouver qu'une faute a été commise. Il ne semble pas y avoir suite à cette promulgation une recrudescence des plaintes adressées aux médiateurs, mais un phénomène intéressant semble émerger. Un nombre plus important de patients rentrent en communication avec le service de médiation pour signaler que la prise en charge ne s'est pas déroulée à la mesure de leurs attentes. Publius Syrius, poète latin et ancien esclave, a écrit : « Supporte sans te plaindre ce qui ne peut être changé », il est donc positif de traduire une plainte comme une interaction positive avec nos patients qui veulent participer à l'amélioration du système de soins.

La gestion systématique des plaintes et l'existence d'une médiation devraient être indissociables afin de combiner efficience et humanisation. Il faut, en effet, assurer une prise en charge de proximité des doléances de tout patient, sans exception. Celui-ci a souvent eu l'impression que l'on s'intéressait certes à sa maladie ou son problème mais pas à sa personne et à ses attentes. Il faut donc recréer un dialogue dès la réception de la plainte en accusant réception de celleci et en assurant qu'un suivi personnalisé va être réalisé. $\mathrm{La}$ politique de réponse se doit d'être systématique et professionnelle, soutenue par le chef de service, les responsables médicaux et infirmiers. L'apport d'un médiateur est majeur pour recréer un lien de confiance entre le patient ou ses proches et le médecin ou l'hôpital. Dans un premier temps, par son écoute attentive de la doléance du patient, il atteste que l'institution et le service sont sensibles tant aux faits reprochés qu'à la composante affective qui met ceux-ci en résonance et en souffrance. Dans un second temps, le médiateur apportera les informations nécessaires au patient sur la suite de la gestion de sa plainte et les possibilités qui sont siennes. Le médiateur restitue alors en interne avec 
objectivité et empathie les difficultés exprimées par le patient. Une réponse écrite et éventuellement une rencontre concluent la démarche de médiation. Il est ensuite de la responsabilité de tout le service d'exploiter le message reçu dans la plainte pour améliorer la démarche de communication et de qualité du service. Dans notre expérience, il n'est pas exceptionnel que le patient exprime que la verbalisation de sa plainte a été un réel soulagement. Fort de cette constatation, certains médecins contactent dès réception de la plainte le patient par téléphone pour une première discussion qui est souvent très positive pour chaque partie.

Certains patients n'accepteront pas la médiation ou resteront insatisfaits. Il n'est pas facile de repérer systématiquement un profil particulier qui sera réfractaire à cette approche.

Parfois, des enjeux identitaires ou familiaux viennent interférer dans cette tentative de résolution de conflit. Les conséquences médicales ou psychologiques d'une prise en charge perfectible sont parfois telles que l'incompréhension ou la colère persiste. Il est rare, heureusement, mais pas impossible que l'échec soit lié à une tentative d'instrumentalisation par le patient pour obtenir un bénéfice secondaire et/ou financier. Dans ce contexte, le médiateur et le chef de service doivent être prêts à proposer, avant la judiciarisation de la situation, d'autres méthodes de résolution des conflits telles que la conciliation ou la transaction avec l'organisme assureur de l'hôpital.

Outre le traitement individuel, il est utile de réaliser une analyse périodique de l'ensemble des plaintes reçues, une base annuelle est recommandée. Cette analyse présente plusieurs avantages à savoir l'identification de plaintes récurrentes portant un sujet identique qui dévoilent alors soit un dysfonctionnement structurel ou organisationnel d'autant plus important qu'il s'avère répétitif, soit un comportement inadéquat d'un ou de plusieurs soignants qui engendrent des plaintes souvent du même ordre (accueil, savoir-être, etc.). Avec ces données, une réelle opportunité existe d'analyser divers critères tels que l'objet, la typologie et le but de la plainte, sa provenance et le mode d'intervention du plaignant, l'importance de son impact sur le devenir $\mathrm{du}$ patient. Il est possible ensuite de définir un niveau de plainte considéré soit comme acceptable, soit comme niveau à atteindre en termes de qualité. Cet indicateur permettra alors la comparaison entre les périodes analysées ou avec d'autres institutions de soins.

Pour conclure, la gestion systématique des plaintes est un moyen unique pour rétablir le lien de confiance entre un patient et l'hôpital. Elle est aussi une réelle opportunité d'améliorer la qualité des soins en faisant tourner sans interruption la « roue de la qualité » de Deming (planifier, développer, contrôler, ajuster) sur les routes et chemins de traverse de notre jeune spécialité.

Remerciements L'auteur tient à exprimer sa gratitude auprès de madame Maria Jesus Alvarez Baranga, médiatrice du service des urgences des cliniques Saint-Luc, auprès de tous ces collaborateurs convaincus de la nécessité d'une démarche qualité, auprès de monsieur Piet Vanormelingen, médiateur institutionnel, et auprès de tous les patients qui nous apprennent chaque jour davantage sur nos pratiques.

\section{Référence}

1. Raynaud-Lambinet A, Juchet H, Charpentier S, et al (2011) Analyse des lettres de plaintes adressées dans un service d'urgence de 2002 à 2007. Ann Fr Med Urgence 1: (in press). 